\title{
OPTIMIZED DESIGN OF THE PRESTRESS IN CONTINUOUS BRIDGE DECKS
}

\author{
M. A. Utrilla and A. Samartín
}

\begin{abstract}
In this paper a method for automatic design of the prestress in continuous bridge decks is presented. In a first step of the procedure the optimal prestressed force for a completely geometrically defined and feasible prestress layout is obtained by means of linear programming techniques. Further on, in a second step the prestress geometry and minimum force are automatically found by steepest descent optimization techniques. Finally this methodology is applied to two-span continuous bridge decks and from the obtained results some preliminary design rules can be drawn.
\end{abstract}

\section{INTRODUCTION}

The design of the layout and forces of the prestress tendons has usually been carried out by the use of heuristic rules and frequently some of these rules were improved by the expertise and intuition of the designer. However, this procedure does not seem to be optimal. Due to the recent computer advances it is possible to use a more direct approach in order to automatically reach the optimal prestress design with minimum designer intervention.

Some previous research efforts in this direction [1-5] allow us to believe in the feasibility of this objective. However, in this work some drastic simplifications such as linearization of the prestress losses, geometry of the tendons, loading and analysis of the stress resultants etc. or some reduction in the number of the variables involved in the problem have been introduced. In some cases the scope of the work is reduced only to the statically determinate structures. Despite the limitations these approaches have often provided quite reasonable results and they were often improved by the designer in a later stage of the process of obtaining the final design.

It is intended in this paper to reach some of the objectives listed below:

- Develop a method to automatically find the prestress force and layout.

- Find the optimal prestress design with reference to an objective function such as minimum weight, minimum amount of prestress, minimum total cost etc.

- Apply the techniques previously developed to two equal-span continuous bridge decks in order to reach some useful prestress design criteria and rules.

\section{HYPOTHESIS}

This study will be developed within the framework of the structural analysis of continuous post-stressed bridge decks under the following assumptions [6]:

(1) The design of the bridge deck is carried out in order to minimize the total prestress cost under the restriction that the working stresses should be within a given range of admissible stresses. Therefore other input design criteria such as ultimate loading, allowable beam deflection, shear contribution, etc. have not been considered in this paper.

(2) The bridge decks have a straight planform and they will be built monolithically in only one construction phase.

(3) The decks will be structurally idealized by a 1-D model composed of straight bars and their analysis will be carried out using the standard matrix structural analysis methods.

(4) The deck will be analysed for the service state (working stresses) under the following loading conditions: dead loads, live loads corresponding to the traffic loads placed in the most critical positions. The corresponding stress resultants will be computed at each cross-section of the structural model.

(5) The prestress design will be checked by the method of allowable stresses. The longitudinal stresses due to the bending moments and the axial forces are the only ones to be considered in this approach.

(6) The prestress is introduced in the bridge deck by means of adherent tendons simultaneously put in tension by jacks at the two ends of each tendon. The prestress frictional losses are computed 
considering the actual geometry of the prestress layout. However the remaining prestress losses are found approximately by the use of a reduction coefficient of the initial prestress force. The prestress secondary effects in the structure, i.e., the redundant forces produced by the static indeterminacy of the bridge deck, have been taken into account throughout all the analysis.

Some of the above-mentioned assumptions can be relaxed without any substantial changes in the methodology to be presented. Then it is possible to consider in this approach other situations such as curved bridge decks, construction in different phases, additional loading conditions (temperatures, settlements), design criteria in shear stresses and ultimate states rather than service states etc.

\section{METHODOLOGY}

The procedure presented here is composed of two models. The first model is in fact embedded into the second one. Both models are briefly described in the following paragraphs.

\subsection{Model 1: Deduction of the optimal prestress force}

The computation of the prestress force is found by solving a linear programming problem [7-14]. In this case the geometry of the tendon elevations is completely defined and only the corresponding tendon force has to be found. At each checking section the stresses due to the external loadings can be computed as well as the ones due to each tendon for a unit prestress force applied at each tendon. At each checking section the total stresses in the bottom and top fibre of the section can be found for the different loading combinations. The constraints of these stresses at each checking section are that they must lie between the allowable stress limits. The use of an algorithm of linear programming can be applied in order to find the tendon forces such that the total tendon forces multiplied by the corresponding tendon lengths are minimized (a value proportional to the total weight of all tendons).

Mathematically the problem to be solved can be expressed as follows.

Find the forces of all tendons contained in the vector $T=\left\{T_{\mathrm{n}}\right\}$, dimension $(N \times 1)$, where $N$ is the number of tendons such that the following objective function is minimized:

$$
\mathrm{OF}=L^{T} T
$$

where $L=\left\{L_{n}\right\}$, is a $(N \times 1)$ dimension vector containing the lengths of the tendons. It is assumed that the geometry of each tendon is completely defined.

The unknown forces vector $T$ must satisfy the following constraints representing the condition that the bottom stresses, $I$, and the top stresses, $S$, at each checking cross-section should lie between the upper and the lower allowable stresses limits for all the different loading conditions during the construction and the service states, i.e.

$$
\begin{aligned}
& \sigma_{-}^{l} \leqslant S_{j}^{\prime} T+S_{o j}^{\prime} \leqslant \sigma_{+}^{\prime} \\
& \sigma_{-}^{\prime} \leqslant T_{j}^{l} T+I_{0 j}^{\prime} \leqslant \sigma_{+}^{\prime}
\end{aligned}
$$

for $j=1,2, \ldots, J$ number of checking cross-sections and $l=1,2,3$ the loading conditions $(l=1$ construction, $l=2$ service with the live load in the position producing the maximum bending moment at the checking section under study and $l=3$ service with the live load in the position producing the minimum bending moment at the checking section under study.

The following notation has been used in eqn (2):

- $\sigma_{-}^{\prime}, \sigma_{+}^{l}$, maximum and minimum allowable stresses for the loading condition $l$.

- $S_{\mathrm{o} j}^{\prime}, I_{\mathrm{o} j}^{\prime}$, stresses at the top fibre, $S$, and the bottom fibre, $I$, of the cross section produced by the external loads in the loading condition, $l$.

- $S_{j}^{\prime}, I_{j}^{\prime}$, are $(1 \times N)$ dimension vectors. The element $n$ of these vectors represents the stresses produced at the top fibre, $S$, or at the bottom fibre, $I$, respectively due to the prestressed tendon $n$ put in tension with a unit force $\left(T_{n}=1\right)$.

Besides the above mechanical restrictions the following constraints must be satisfied:

$$
T_{n} \geqslant 0
$$

\subsection{Model 2: Automatic generation of the force and geometry of the optimal prestress}

In this model the geometry and prestress force are both simultaneously found by the use of nonlinear optimization techniques, namely the steepest descent gradient $[15,16]$.

The model starts from a given feasible prestress layout defined by a set of singular points. Each of these points is defined by three coordinates $\left(x_{m}, y_{m}, \theta_{m}=\mathrm{d} y / \mathrm{d} x\right)$. Between two consecutive singular points a cubic hermitian polynomial is interpolated and the tendon elevation is in this way completely defined. Using the methodology of Model 1 the optimal prestress force corresponding to this tendon geometry is found. The changes in the initial prestress layout are introduced iteratively by computing numerically the gradient of the objective function.

In mathematical terms the above procedure is described as follows. The geometry of a generic tendon $n$ is described by a set of singular points or parameters vector $q_{m}^{n}=\left(x_{m}, y_{m}, \theta_{m}\right)$ ordered in such a way that $x_{m}^{n}<x_{m+1}^{n}$. Between two consecutive parameters vector, $q_{m}^{n}$ and $q_{m+1}^{n}$, a cubic hermitian 
polynomial is interpolated. That means the whole tendon geometry $y_{n}(x)$ can be described by a set of piecewise polynomials containing a total number of $3 \times M_{n}$ parameters

$$
q_{m}^{n}=\left(x_{m}, y_{m}, \theta_{m}\right)
$$

where

$$
m=1,2,3, \ldots, M_{n} .
$$

Some values of these parameters are specified constants due to project requirements (for example tendon anchorage points) and the remaining ones are unknowns to be determined, and these parameters will be called active parameters or active degrees of freedom. All these active dof will be kept in a single vector $\tilde{q}^{n}$.

Therefore the problem to be solved is stated as: to find the vector of active dof $\tilde{q}^{n}$ and the values of the prestress forces $T=\left\{T_{n}\right\}$ for each tendon $n$ $(n=1,2, \ldots, N)$, in such a way that the objective function eqn (1) be minimized and the mechanical constraints eqns (2) and (3) be satisfied.

Apart from the above mechanical constraints there exists in this model constraints related to the geometry of the prestress. These geometrical constraints can be expressed at each abscise $x$ of the cable $n$ as follows:

$$
\begin{gathered}
y_{\min }(x) \leqslant y_{n}(x) \leqslant y_{\max }(x) \\
\left|\frac{\mathrm{d}^{2} y_{n}(x)}{\mathrm{d} x^{2}}\right| \leqslant \frac{1}{R}
\end{gathered}
$$

The minimum cover at the top and bottom faces of the section for each tendon is expressed by conditions of eqn (4) and the minimum value for the curvature radius of the tendon is introduced by eqn (5).

The problem just stated is a highly nonlinear one: note now that $L$ depends on the parameters $\tilde{q}^{n}$ and the following iterative procedure has been used in order to solve it.

Start with a geometrically admissible layout of the prestress tendons, i.e. a prestress geometry satisfying the conditions of eqns (4) and (5). For this case Model 1 allows us to find the values of the prestress forces $T$ by solving a linear programming problem. From the known prestress geometry and forces it is possible to evaluate the objective function of the corresponding problem $\mathrm{OF}=L^{\top} T=\mathrm{OF}\left(\tilde{q}^{n}\right)$ that depends only on the active dof because $L$ as well as $T$ can be expressed in terms of these active dof.

It is possible to modify the prestress geometry to a new one nearer to the optimal geometry by using an algorithm of the steepest gradient type. This algorithm is described as follows.

It is assumed that in the current iterative computational step $k$ the value of the objective function $\mathrm{OF}^{\mathrm{k}}=\mathrm{OF}\left(\tilde{q}_{\mathrm{k}}\right)$ is known for a given set of determinate values of the active parameters of all prestress tendons contained in the parameter vector $\tilde{q}_{k}=\left\{\tilde{q}_{k}^{1}, \tilde{q}_{k}^{2}, \ldots, \tilde{q}_{k}^{N}\right\}$ where $\tilde{q}_{k}^{n}$ represents the vector of the current values of these dof of the tendon $n$ at the computational step $k$.

The gradient of the objective function $\mathrm{OF}^{k}$ is computed numerically by giving a small increment to each active dof $\Delta \tilde{q}$ i.e. the following ratios defining the objective function gradient are computed:

$$
\nabla \mathrm{OF}=\frac{\mathrm{OF}\left(\tilde{q}_{k}+\Delta \tilde{q}\right)-\mathrm{OF}\left(\tilde{q}_{k}-\Delta \tilde{q}\right)}{2 \Delta \tilde{q}}
$$

The new prestress geometry defined by the active parameters vector $\left\{\tilde{q}_{k+1}\right\}$, is determined by the expression:

$$
\tilde{q}_{k+1}=\tilde{q}_{k}+\lambda \nabla O \mathrm{~F}_{k}
$$

where

$$
\lambda=\frac{\Delta \tilde{q}}{\max \{\nabla O F\}} .
$$

Before the admission of the prestress layout given in eqn (7) it is necessary to check its geometrical validity i.e. if the geometrical constraints of eqn (4) and (5) are fulfilled.

Similarly during the computation of the gradient of the objective function it is important to check the geometrical validity of the tendon layout associated with the increment of the dof. In the condition that this requirement is not satisfied, the corresponding increment of the dof under consideration cannot take place and it is assumed to be null.

The new geometric prestress layout values represent the initial prestress geometry to start the next computational iterative step $k+1$.

This procedure goes on until an acceptable convergence is reached, i.e. the condition $\left|O F^{k+1}-O F^{k}\right| \leqslant \epsilon$ or a maximum specified number of iterations has been surpassed.

Using the described procedure it is possible to change an initially given prestress geometry towards a new one nearer to the optimal prestress geometry and with the minimum value of the prestress force in each geometry to satisfy the mechanical constraints. That means that for a new computational step the following condition holds:

$$
\mathrm{OF}^{k+1} \leqslant \mathrm{OF}^{k}
$$

In order to reduce the computational effort the gradient is kept constant throughout several computational steps until the value of the objective function in the step $k+1$ is greater than that of the objective 
function of the previous step $k$. In this case the gradient should be updated at step $k$.

\section{DETERMINATION OF AN INITIAL FEASIBLE SOLUTION}

The application of the procedure described in the preceding section demands the prior knowledge of a mechanical as well as geometrically feasible solution, i.e. a prestress layout and forces for all tendons fulfilling the geometrical constraints of eqns (4) and (5) and producing admissible stresses or equivalently stresses satisfying the conditions of eqns (2) and (3) at all checking cross-sections and must achieve this for all loading conditions.

Usually an initial feasible prestress geometry is efficiently designed by following some heuristic design rules. However, there exist some design situations, with special loading conditions or structural singularities for which these simple design rules cannot be applied. Moreover, in many cases the dimensions of the concrete cross-sections or the concrete strength are not sufficient to resist the final stresses. In these cases it is necessary to apply a general tool in order to find automatically a feasible solution and if such a feasible solution does not exist to obtain an indication of this fact.

The proposed method to find a feasible initial solution is summarized as follows.

Construct a geometrical feasible solution by means of empirical design rules (covers and curvature restrictions must be considered). In general, the resulting stresses from this empirical design will not fulfil the mechanical constraints i.e. they will not produce admissible stresses at each checking crosssection $j$.

Defining the following objective function:

$$
\mathrm{OFR}=\sum_{i=0}^{i=R} \epsilon_{i}
$$

where $R$ is the total number of constraints equations given by all the constraints, eqn (2). The error $\epsilon_{i}$ is defined as follows: Constraints conditions type $A_{i} \geqslant 0$ where:

$$
A_{i}=\bar{\sigma}_{i} T+\sigma_{i}^{\prime}-\sigma_{-}^{\prime}
$$

then:

$$
\begin{gathered}
\epsilon_{i}=0, \text { if } A_{i} \geqslant 0 \\
\epsilon_{i}=-A_{i} \text { if } A_{i}<0
\end{gathered}
$$

Restraints conditions type $B_{i} \leqslant 0$ where:

$$
B_{i}=\bar{\sigma}_{i} T+\sigma_{i}^{\prime}-\sigma_{+}^{\prime}
$$

then:

$$
\begin{array}{cc}
\epsilon_{i}=B_{i}, \quad \text { if } \quad B_{i} \geqslant 0 \\
\epsilon_{i}=0 \text { if } \quad B_{i} \leqslant 0
\end{array}
$$

For a feasible prestress the function OFR given by eqn (9) will be null, i.e. all the mechanical constraints are satisfied.

Therefore it is possible to find an initial feasible solution from a geometrical feasible solution by solving the following optimization problem: $\mathrm{min}$ OFR, for all the sets of the design variables

$$
T y q_{m}^{n}=\left(x_{m}, y_{m}, \theta_{m}\right)
$$

It is possible to set up this optimization problem to be solved by linear programming techniques or in an unrestrained nonlinear optimization problem by a penalty technique [17]. However, these alternative formulations will not be presented here. The solution procedure used to solve the above problem which has been described in the previous section is the steepest gradient method.

If the minimum value of the OFR is significantly different from zero then a feasible solution cannot be found because it does not exist. That means that the basic design assumptions about the design should be modified (such as the cross-sectional dimensions, strength of the concrete etc.).

\section{APPLICATION TO BRIDGE DECKS WITH TWO EQUAL SPANS}

The methodology developed in the previous sections has been applied to the particular case of a set of continuous bridge decks with two equal spans.

The geometric definition of the bridge deck and the main variables used in its definition are shown in Fig. 1. The deck is modelled as a continuous beam of two equal spans and constant depth. Two types of transversal cross-sections are considered: solid and voided cross-sections.

The following range for the dimensions of this type of bridge decks has been considered:

- Span: $L=$ from $15.00 \mathrm{~m}$ up to $40.00 \mathrm{~m}$ with step $\Delta L=5.00 \mathrm{~m}$.

- Width: $B=$ from $8.00 \mathrm{~m}$ up to $16.00 \mathrm{~m}$ with step $\Delta B=4.00 \mathrm{~m}$.

- Depth: $H=L / \lambda$ where the slenderness $\lambda$ varies from 18 up to 30 with step $\Delta \lambda=2$.

These sets of bridge decks represent 126 cases to be analysed. All the decks are assumed to be built in prestressed concrete with characteristic strength of 


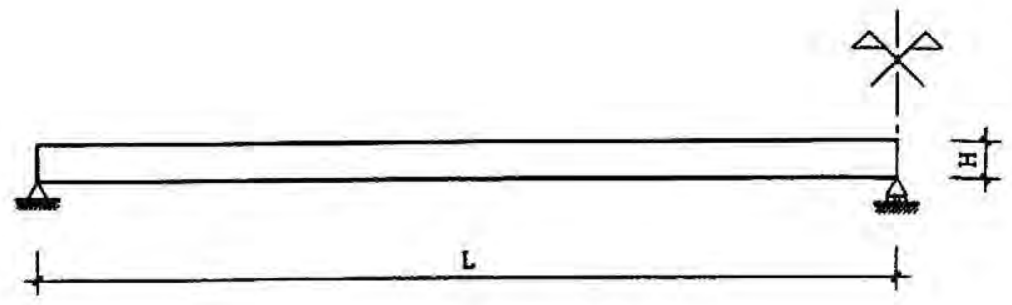

(a) LONGITUDINAL SECTION



(b) SOLID

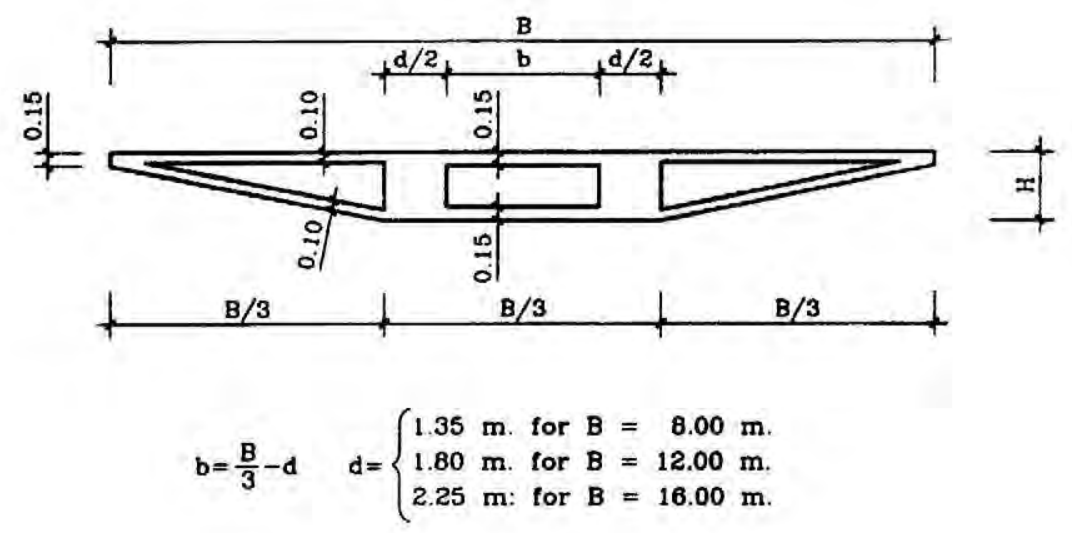

(c) VOIDED

$\mathrm{L}=$ Span

$\mathrm{B}=$ Width

$H=$ Depth.

Fig. 1. Geometry and notation.

$350 \mathrm{kp} / \mathrm{cm}^{2}$, and that represents the following interval of allowable stresses:

- Compression: $\sigma_{+}=219 \mathrm{kp} / \mathrm{cm}^{2}$.

- Tension: $\sigma_{-}=-20 \mathrm{kp} / \mathrm{cm}^{2}$.

In Fig. 2 the different loads to be applied to the bridge decks are shown.

In order to reduce the total computational time, all prestress tendons are reduced to only one mean tendon. The mean tendon is symmetric with respect to the central support and it can be defined by $3 \times 7=21$ dofs. However, due to the already mentioned symmetry and obvious design constraints the number of active dof is reduced to $8,\left(y_{1}, \theta_{1}, x_{2} y_{2}, x_{3} y_{3} \theta_{3}, y_{4}\right)$, according to Fig. 3. The geometry of this mean tendon between two consecutive singular points is approximated 


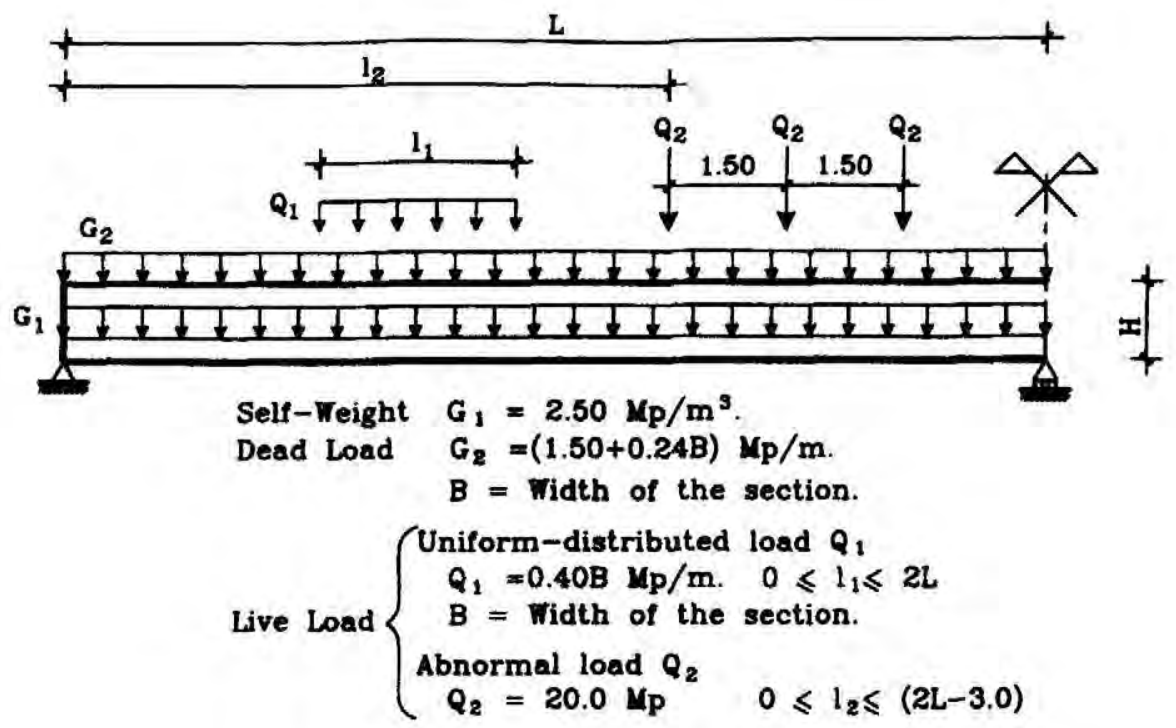

Fig. 2. Loads.

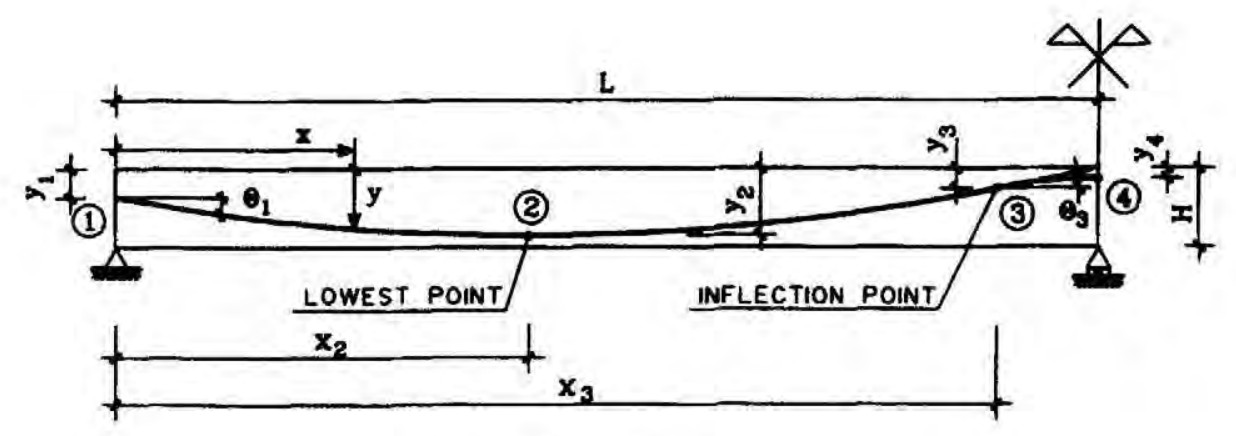

\section{DESIGN CONSTANTS}

PRESTRESS LOSSES

$$
\left\{\begin{array}{l}
\text { Frictional loss : } T=\text { To } e^{-(\mu \alpha+k x)} \\
\mu=0.21 \text { Curvature friction coefficient. } \\
k / \mu=0.006 \quad k=\text { Wobble friction coefficient. } \\
\alpha=\text { Angular variation. } \\
\text { Initial losses }=5 \pi+\text { frictional loss. } \\
\text { Total losses }=15 \%+\text { frictional loss. }
\end{array}\right.
$$

REC $<y<H-$ REC $\quad$ REC $=0.15 \mathrm{~m}$. (minimum cover).

Minimum radius $(R)=5.00 \mathrm{~m}$.

$$
\begin{aligned}
& \text { DEGRESS OF FREEDOM }
\end{aligned}
$$

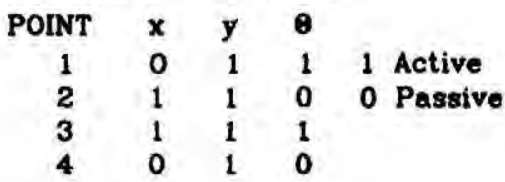

Fig. 3. Prestress cable profile. 
by a cubic hermitian polynomial, as has already been discussed. In Fig. 3 the values of some design constants, such as the friction coefficient, minimum cover and curvature radius etc., are also included.

\section{DISCUSSION OF THE RESULTS}

Some conclusions about the optimal design obtained from the results computed for the different bridge decks analysed in Section 5 can be drawn.

The prestress layout producing the minimum prestress force can be approximated by the following expressions that have been found by a linear regression through the values computed for the 126 bridge decks.

\subsection{Voided section}

Horizontal position of the singular points of the prestress cable profile (Fig. 3).

Lowest point:

$$
\frac{X_{2}}{L}=0.407 \pm 0.013
$$

Inflection point:

$$
\frac{L-X_{3}}{L}=0.098 \pm 0.012
$$

Vertical position of the singular points of the prestress cable profile (Fig. 3).

Lowest point:

$$
\frac{Y_{2}-V_{8}}{V_{1}-\mathrm{REC}}=1.000 \pm 0.002
$$

Inflection point:

$$
\frac{V_{\mathrm{s}}-Y_{4}}{V_{\mathrm{s}}-\mathrm{REC}}=1.000 \pm 0.000
$$

where $V_{\mathrm{s}}$ and $V_{\mathrm{i}}$ are the distances of the centroid of the cross-section to the top and bottom faces of the bridge deck.

\subsection{Solid section}

Horizontal position of the singular points of the prestress cable profile (Fig. 3).

Lowest point:

$$
\frac{X_{2}}{L}=0.409 \pm 0.019
$$

Inflection point:

$$
\frac{L-X_{3}}{L}=0.087 \pm 0.013
$$

Vertical position of the singular points of the prestress cable profile (Fig. 3).

Lowest point:

$$
\frac{Y_{2}-V_{\mathrm{s}}}{V_{i}-\mathrm{REC}}=0.999 \pm 0.002
$$

Inflection point:

$$
\frac{V_{s}-Y_{4}}{V_{s}-\text { REC }}=1.000 \pm 0.000
$$

It can be observed from the above expressions that cable layout for both types of sections, producing the minimum cost in a two-span continuous bridge and under the assumptions given in the paper, is as follows:

(1) The lowest point of the cable should be located at the section distant from the extreme support 0.40 times the span length. The ordinate of the cable at this section should be maximum, i.e., the depth bridge minus the cover.

(2) The ordinate of the cable at the section of the intermediate support should be minimum, i.e, equal to the cover.

(3) The inflection point of the cable should be situated at the section distant from the intermediated support 0.10 times the span length. The ordinate of the cable at this section is automatically determinate by simple geometrical considerations from the lowest and the highest ordinates of the cable.

In order to approximate to a real optimal design problem that includes the costs of the concrete as well as the costs of the passive and active steel in a total bridge deck an estimation has been carried out of the total deck costs $(C)$. The unitary prices used in the material construction costs are given in Table 1.

Table 1. Cost per unit (pesetas)

\begin{tabular}{lrr}
\hline Units & $\begin{array}{r}\text { Voided } \\
\text { section }\end{array}$ & $\begin{array}{r}\text { Solid } \\
\text { section }\end{array}$ \\
\hline $\mathrm{m}^{3}$ of concrete & $14,000.0$ & $12,000.0$ \\
$\mathrm{~m}^{3}$ of centring & 1000.0 & 1000.0 \\
$\mathrm{~m}^{2}$ of seen shuttering & 3500.0 & 3500.0 \\
$\mathrm{~m}^{2}$ of hidden shuttering & 2500.0 & 2500.0 \\
$\mathrm{~K}$ p of steel for reinforced & 110.0 & 110.0 \\
$\mathrm{~K}$ p of steel for prestressed & 350.0 & 350.0 \\
\hline
\end{tabular}


Table 2. Numerical results for voided and solid sections

\begin{tabular}{|c|c|c|c|c|c|c|c|}
\hline \multirow{2}{*}{$\begin{array}{l}L \\
(\mathrm{~m})\end{array}$} & \multirow{2}{*}{$\begin{array}{c}B \\
(\mathrm{~m})\end{array}$} & \multicolumn{2}{|c|}{$\lambda_{\min }$} & \multicolumn{2}{|c|}{$Q_{\min }\left(\mathrm{K} \mathrm{p} / \mathrm{m}^{2}\right)$} & \multicolumn{2}{|c|}{$C_{\min }\left(10^{3} \mathrm{pts}\right)$} \\
\hline & & V.S. & S.S. & V.S. & S.S. & V.S. & S.S. \\
\hline 15.00 & 8.00 & 20.63 & 22.77 & 7.42 & 11.69 & 26.38 & 25.06 \\
\hline 15.00 & 12.00 & 21.22 & 23.70 & 6.30 & 10.74 & 25.62 & 24.44 \\
\hline 15.00 & 16.00 & 21.35 & 24.30 & 5.67 & 10.23 & 25.26 & 24.10 \\
\hline 20.00 & 8.00 & 23.30 & 25.40 & 10.28 & 16.90 & 28.13 & 27.94 \\
\hline 20.00 & 12.00 & 22.90 & 26.49 & 8.52 & 15.73 & 27.21 & 27.18 \\
\hline 20.00 & 16.00 & 22.80 & 26.93 & 7.67 & 15.02 & 26.74 & 26.79 \\
\hline 25.00 & 8.00 & 24.40 & 27.08 & 12.88 & 22.33 & 29.88 & 30.97 \\
\hline 25.00 & 12.00 & 24.00 & 27.92 & 10.76 & 20.87 & 28.69 & 30.14 \\
\hline 25.00 & 16.00 & 23.72 & 28.45 & 9.74 & 20.13 & 28,14 & 29.71 \\
\hline 30.00 & 8.00 & 25.33 & 28.31 & 15.64 & 28.45 & 31.61 & 34.26 \\
\hline 30.00 & 12.00 & 24.85 & 28.67 & 13.27 & 26.50 & 30.22 & 33.36 \\
\hline 30.00 & 16.00 & 24.36 & 28.87 & 11.93 & 25.52 & 29.52 & 32.92 \\
\hline 35.00 & 8.00 & 25.93 & 28.78 & 18.51 & 34.83 & 33.39 & 37.81 \\
\hline 35.00 & 12.00 & 25.29 & 29.19 & 15.73 & 32.84 & 31.73 & 36.85 \\
\hline 35.00 & 16.00 & 24.94 & 29.53 & 14.39 & 31.96 & 30.94 & 36.39 \\
\hline 40.00 & 8.00 & 26.71 & 29.36 & 21.78 & 42.19 & 35.21 & 41.65 \\
\hline 40.00 & 12.00 & 25.73 & 29.83 & 18.48 & 40.17 & 33.32 & 40.62 \\
\hline 40.00 & 16.00 & 25.37 & 29.97 & 16.97 & 39.09 & 32.39 & 40.13 \\
\hline
\end{tabular}

An average ratio of passive reinforcement which has been well established in professional practice giving the ratio of weight of reinforcement to deck platform area of $50 \mathrm{kp} / \mathrm{m}^{2}$ has been used.

For every deck with a fixed span, $L$, and width, $B$, the values of the depth and prestress force producing the minimum total deck cost are determinate. They will be denoted by $H_{\min }$ and $T_{\min }$ and the corresponding minimum cost per unit of deck area by $C_{\min }$. Tables 2 and 3 contain these values as functions of the span length and the width of the deck. In fact the values used in the tables are the slenderness $\left(\lambda_{\min }=L / H_{\min }\right)$ and the prestress weight per unit of area of the deck planform $\left(Q_{\min }\right)$.

From the above results it is observed that the self weight has an important role in the total cost of the bridge deck. Therefore it is of great interest to try to reduce the volume of concrete in the deck to feasible constructional limits.

Table 3. Voided section $\left(\epsilon_{\lambda}=0.13 ; \epsilon_{\mathrm{Q}}=0.05 ; \epsilon_{\mathrm{C}}=0.02\right)$

\begin{tabular}{lrrr}
\hline \multicolumn{4}{c}{ Coefficient $a_{i j}$ for values of $j$} \\
\hline$i$ & 0 & 1 & \multicolumn{1}{c}{2} \\
\hline 0 & 5.58052349 & 1.09098543 & -0.02627516 \\
1 & 1.11280645 & -0.06571114 & 0.00136573 \\
2 & -0.01276277 & 0.00063590 & -0.00000806 \\
\hline \multicolumn{4}{c}{ Coefficient $b_{i j}$ for values of $j$} \\
\hline$i$ & \multicolumn{4}{c}{1} \\
\hline 0 & 0 & -0.04346957 & 0.00436107 \\
1 & 0.42458893 & -0.03944852 & 0.00076681 \\
2 & 0.00282492 & -0.00016861 & 0.00001195 \\
\hline \multicolumn{4}{c}{ Coefficient $c_{i j}$ for values of $j$} \\
\hline$i$ & \multicolumn{4}{c}{1} \\
\hline 0 & 22.26475444 & -0.15481530 & 0.00478290 \\
1 & 0.43033202 & -0.01557865 & 0.00040687 \\
2 & 0.00154022 & -0.00019340 & 0.00000596 \\
\hline
\end{tabular}

The results of Table 2 can be expressed in analytical form according to the following polynomial expressions:

$$
\begin{gathered}
\lambda_{\text {min }}=\lambda(L, B)=a_{0}(B)+a_{1}(B) L+a_{2}(B) \mathrm{L}^{2} \pm \epsilon_{\lambda} \\
Q_{\text {min }}=Q(L, B)=b_{0}(B)+b_{1}(B) L+b_{2}(B) \mathrm{L}^{2} \pm \epsilon_{Q} \\
C_{\text {min }}=C(L, B)=c_{0}(B)+c_{1}(B) L+c_{2}(B) L^{2} \pm \epsilon_{i}
\end{gathered}
$$

where

$$
\begin{aligned}
& a_{0}(B)=a_{00}+a_{01} B+a_{02} B^{2} \\
& a_{1}(B)=a_{10}+a_{11} B+a_{12} B^{2} \\
& a_{2}(B)=a_{20}+a_{21} B+a_{22} B^{2}
\end{aligned}
$$

Table 4. Solid section $\left(\epsilon_{i}=0.22 ; \epsilon_{\mathrm{Q}}=0.12 ; \epsilon_{c}=0.01\right)$

\begin{tabular}{lcrr}
\hline \multicolumn{4}{c}{ Coefficient $a_{i j}$ for values of $j$} \\
\hline$i$ & 0 & 1 & \multicolumn{1}{c}{2} \\
\hline 0 & 6.49034817 & 0.98561735 & -0.02741859 \\
1 & 1.05042538 & -0.03375970 & 0.00103110 \\
2 & -0.01292273 & 0.00036857 & -0.00001270 \\
\hline \multicolumn{4}{c}{ Coefficient $b_{y}$ for values of $j$} \\
\hline$i$ & 0 & 1 & 2 \\
\hline 0 & -3.72023195 & 0.61316746 & -0.02203364 \\
1 & 1.15756951 & -0.08604127 & 0.00272140 \\
2 & 0.00417663 & 0.00104970 & -0.00003446 \\
\hline \multicolumn{4}{c}{ Coefficient $c_{i j}$ for values of $j$} \\
\hline$i$ & \multicolumn{4}{c}{1} \\
\hline 0 & 18.59716954 & -0.06569049 & -0.00053209 \\
1 & 0.51969065 & -0.02134024 & 0.00066467 \\
2 & 0.00366197 & 0.00020968 & -0.00000663 \\
\hline
\end{tabular}




$$
\begin{aligned}
& b_{0}(B)=b_{00}+b_{01} B+b_{02} B^{2} \\
& b_{1}(B)=b_{10}+b_{11} B+b_{12} B^{2} \\
& b_{2}(B)=b_{20}+b_{21} B+b_{22} B^{2} \\
& c_{0}(B)=c_{00}+c_{01} B+c_{02} B^{2} \\
& c_{1}(B)=c_{10}+c_{11} B+c_{12} B^{2} \\
& c_{2}(B)=c_{20}+c_{21} B+c_{22} B^{2}
\end{aligned}
$$

In Tables 3 and 4 the coefficients of the formulae of eqns (13)-(15) are given.

In Figs 4 to 6 the curves representing eqns (10)(12) are given for the different widths considered and for the two cases of cross-sections (voided and solid sections).

The conversion factors between the units of the International System (SI) and the units of the system (15) Meter-kilopond-second are as follows:

$1 \mathrm{~N}=0.1102 \mathrm{kp}$
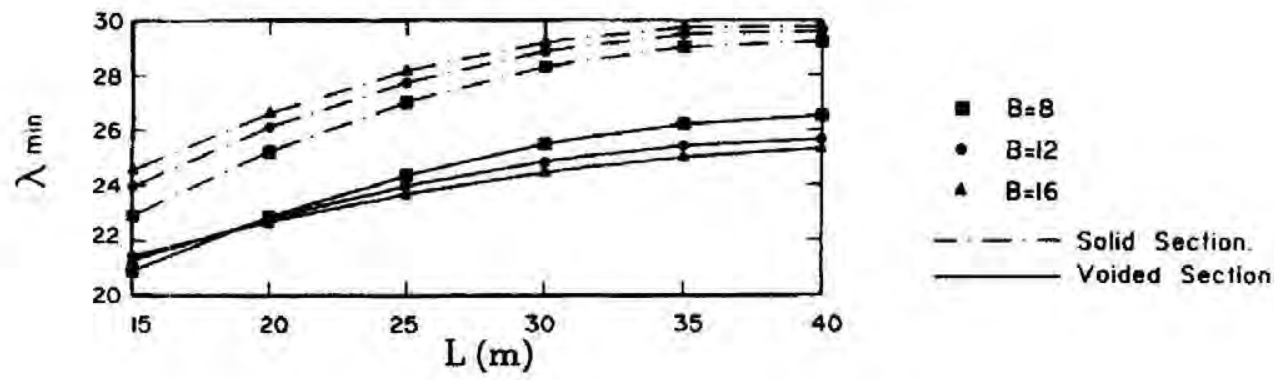

Fig. 4. Slenderness $\left(\lambda_{\text {min }}\right)-$ span length $(L)$.

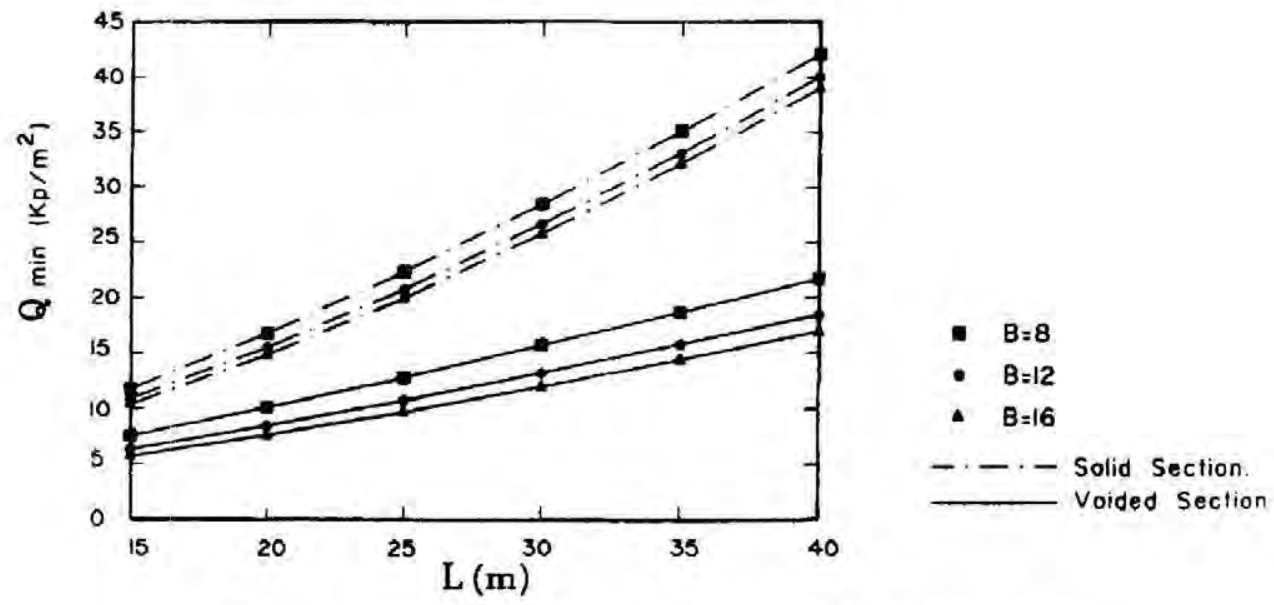

Fig. 5. Prestress weight per unit of deck area $\left(Q_{\min }\right)-$ span length $(L)$.

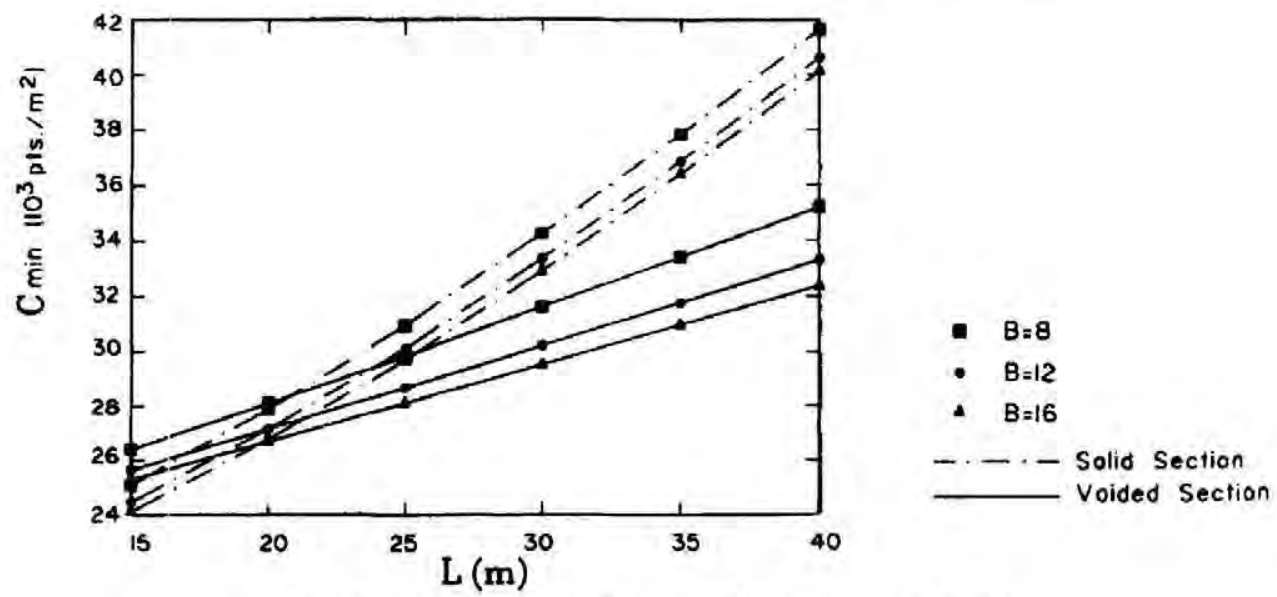

Fig. 6. Cost per unit of deck area $\left(C_{\min }\right)-$ span length $(L)$. 
$1 \mathrm{kp}=9.8 \mathrm{~N}$

$1 \mathrm{~N} / \mathrm{mm}^{2}=1 \mathrm{MPa}=10.2 \mathrm{kp} / \mathrm{cm}^{2}$

$1 \mathrm{kp} / \mathrm{cm}^{2}=0.098 \mathrm{~N} / \mathrm{mm}^{2}$

Acknowledgements - The authors acknowledge the referees' comments that have improved the quality of the paper.

\section{REFERENCES}

I. Maquoi, R. and RandaI, J, Approche realiste du dimensionnement optimal des ponts precointraints hyperstatiques, Ann. Trav. Pub. Belgium, 1977.

2. Maquoi, R. and Randal, J., "Optimal layout of cables in prestressed indeterminate bridges", Revue Roumaine de Sciences Techniques.

3. Kirsch, V., "Optimized prestressing by linear programming", Int. J. Numerical Methods in Engineering, 1973, 7.

4. Kirsch, V., "Optimum design of prestressed beams", Computers and Structures, 1972.

5. González, A., "Método para el dimensionamiento directo del pretensado en puentes hiperestáticos", tesis doctoral. E.T.S.I.C., C. y P. Universidad Politécnica. Madrid, 1989.

6. Samartín, A., Cáculo de estrcturas de puentes de hormigón, (Ed. Rueda) Madrid, 1982.

7. Hernández Ibañez, S., "Métodos de diseño óptimo de estructuras", Colegio de I.C.C. y P. Madrid, 1990.
8. Templeman, A. B. and Winterbottom, S. K., "Optimum design of concrete cellular spine beam bridge decks", Proc. Instn. Civ. Engrs. Parr 2, June 1979, 67.

9. Puy, J., "Programación lineal" Monografias de la Cátedra de Matemáticas III. Universidad Politécnica de Madrid, E.T.S.I.C., C. y P., 1983.

10. Pardo, L., Programacion Lineal Continua (Ed. Diaz de Santos), Madrid, 1987.

11. Pike, R. and Guerra, L., Optimizatión en Ingenieria (Ed. Alfaomega), México, D.F., 1989.

12. Puy, J., "Algoritmos numéricos en Paseal", Servicio de Publicaciones Revista de Obras Públicas, Universidad Politécnica de Madrid, E.T.S.I.C., C. y P., 1985.

13. Nering, E. and Tucker, A., Linear Programs and Related Problems, Academic Press, Inc. San Diego, 1993.

14. Samartin, A. and Utrilla, M. A., "Otpimization of prestressed concrete bridge decks", Computers \& Structures, 1991, 41 (3), 553-559.

15. Radic, D. Dvornik, J. Candrlic, V. Dekanovic, D. and Ozbolt, J., "Optimization of continuous prestressed beams for different construction stages", Gradevinski Institut. Yugoslavia.

16. Oliveira Fernandes de Almeida, J. C., "Dimensionamento das armadures de flexao em vigas contínuas de betao armado pré-esforçao", tesis doctoral, Departamento de Engenharia Civil del I.S.T de la U.T, de Lisboa, 1985.

17. Private communication, Puy, J., E.T.S. de I. de C., C. y P. de la Universidad Politécnica de Madrid. 